\title{
Review of: "Impaired in vitro Interferon-y production in patients with visceral leishmaniasis is improved by inhibition of PD1/PDL-1 ligation"
}

Erika van den Bogaart

Potential competing interests: The author(s) declared that no potential competing interests exist.

The paper authored by Takele et al. presents the results of an immunological study conducted on samples obtained cross-sectionally from a small cohort of VL patients and healthy controls living in Ethiopia. The study investigates the interaction between the immune checkpoint PD1 and its ligand PDL-1 and its effect on the impaired production of IFN-gamma by whole blood cells from VL patients.

The study is scientifically sound as it makes use of an appropriate experimental design to address a relevant research question. Whilst the PD-1/PD-L1 pathway is known to likely regulate immunity against Leishmania parasites, it is not yet fully clear how this immune checkpoint works to control parasite proliferation. The evidence is presented in a clear and logical fashion and is adequate to support the authors' conclusions. Yet, there is a number of queries/comments mainly relating to the methods section that the authours may consider addressing.

1. There are no considerations to justify the sample size nor the choice of recruiting only male subjects.

2. The subjects were recruited in an area with a very high prevalence of HIV-VL co-infections. Were the recruited patients tested for HIV infection? HIV is known to exacerbate immunosuppression in VL patients and may therefore affects the observations made by the authors.

3. It appears that the present study is part of a larger cross-sectional study conducted in the same area and described in reference 14 (same ethical approval numbers). Should this be the case, how were the 16 patients and the 10 controls included in the present study selected from the ones recruited in the larger study (50 cases and 25 controls)? Were they sequentially recruited? I would recommend specify this to exclude any possible bias in the subject selection.

4. As for the previous comment, only the samples of 8 cases (out of the 16 recruited) were investigated for the production of IFN-gamma following the interference with the PD1/PDL-1 ligation (Fig. 4). What was the reason for selecting these 8 samples and how were they selected?

5. More details on the experimental methods would be useful to fully appreciate the adequacy of the experiments performed, including the number of replicates, the type of controls used, how data were analyzed (e.g. for ELISA), etc.

6. There is a subtantial similarity between the first part of the introduction of the present paper and the one of reference 14 . 
